\title{
IoT enabled Plant Sensing Systems for Small and Large Scale Automated Horticultural Monitoring
}

\author{
Sherjeel Khan \\ mmh labs \\ King Abdullah University of Science and Technology \\ Thuwal, Saudi Arabia \\ ORCID ID: 0000-0001-6730-4330
}

\author{
Muhammad Mustafa Hussain \\ mmh labs \\ King Abdullah University of Science and Technology \\ Thuwal, Saudi Arabia \\ ORCID ID: 0000-0003-3279-044
}

\begin{abstract}
We report small scale and large scale sensory systems that can monitor climatic conditions in greenhouses, laboratories, or crops that affect plant growth. An ultralightweight flexible sensory platform using bare die CMOS chips having a light, temperature, and humidity sensor on a flexible polymer substrate is demonstrated. It is made up of flexible and transparent materials and weighs merely 0.44 gram. Thus, the standalone multisensory platform can be seamlessly placed on a plant leaf without affecting its functions. Furthermore, a strain sensor using our unique fabrication strategy with 10 fold enhanced linear strain range (22\%; conventional metal-based strain gauges have $\mathbf{2 - 5 \%}$ linear range) is used to monitor physical plant growth over long time intervals. A framework is further provided for large-scale deployment of these sensors for remote monitoring of large cultivated areas of crops. The sensory platform is equipped with biodegradable paper wings to soften the landing during their deployment, dropped using a compact dropping mechanism. The sensors are equipped with IoT enabled electronics to allow realtime online data representation and monitoring.
\end{abstract}

Keywords—plants; microclimate; sensors; bare-die; lightweight;

\section{INTRODUCTION}

The sharp rise in the global population has put pressure on the agriculture industry to sustain the food supply. With the advent of modern technology and the rising interest in the Internet of Things (IoT) enabled devices, there has been a boom in automation of horticultural production. Photosynthesis is the process that generates food for the plants by converting carbon dioxide and water into sugars. The quality and quantity of food extracted from planted crops depend upon a variety of factors that affect the process of photosynthesis including humidity, light and temperature levels [1]. These factors have a direct effect on the growth of the plant, and thus, consequently, dictate the consumable crop yield from a given area of cultivated land. Crops are usually distributed along hectares of land, which makes it difficult for the farmers to manually monitor the microclimatic conditions in different areas of the cultivated field. Current techniques use drones equipped with highresolution cameras to obtain a heat map of crops from the air. However, such methods have been shown to be inconclusive as false readings and failure rates are predominant due to complex topographical landscapes, and limitations of the image processing methods used [2]. Moreover, such methods are unable to detect the humidity and light levels in different areas of the field from such a distance. The challenges are brought by the fact that the drone needs to analyze a crop field from a long distance. There is a need for a method of monitoring microclimatic conditions in crops at a ground level. Due to the vast scale of cultivated lands, it is almost impossible to undertake this task through manual human in-field retrieval.

Even at a smaller level inside a greenhouse, the current techniques are inefficient to provide information about the actual climate experienced by the plants. Centralized climate monitoring devices, which are sometimes placed in the soil around the plants fail to capture the actual microclimate conditions seen by a plant leaves as the photosynthesis process takes place in the leaf $[3,4]$. Sensors placed in the soil next to the plant cannot accurately show the temperature, humidity, and light experienced by the plant. Plants are regularly irrigated or watered. The water absorbed by the soil affects the humidity and temperature readings from sensors placed in the soil. The light reaching the sensor is different from the light seen by the plant as the sensor lies in the shadow of the plant itself. There is a clear demand for systems that can monitor the microclimate conditions around the leaf of the plants. This can be achieved by placing sensors on the leaf itself. However, due to the bulky electronic interface system, most leaf based sensory systems have the interface electronics present next to the plant and the sensors placed on the leaf are connected via wires [5-7]. The wires interfere with the growth of the plant itself.

In this paper, we present solutions for both microclimate monitoring at a smaller level using ultra-lightweight flexible and transparent sensory platform that can be placed on the leaf of a plant for microclimate monitoring[8] and a strategy for large-scale deployment strategy of multisensory platforms for remote macroclimate sensing of large-scale cultivated lands. The ultra-lightweight flexible sensory platform contains flexible light, temperature, and humidity sensors interfaced with electronics and a battery, with a net weight of 0.44 grams. The transparent lightweight platform can be placed on the leaf without affecting its growth. In addition, we present plant growth monitoring sensors using flexible strain sensors that can monitor plant growth at a micrometer level [9]. We have shown that the add-on approach enhances the functionality of existing things non-intrusively for marine species and a prescription bottle [10-12]. For the large-scale deployment, we have further developed flexible lightweight sensors that can be deployed from a drone. The flexible sensors are wrapped around a small battery equipped with wings made from biodegradable paper to soften the landing when launched from a drone using a specially designed dropping mechanism. The drone can take a reading 


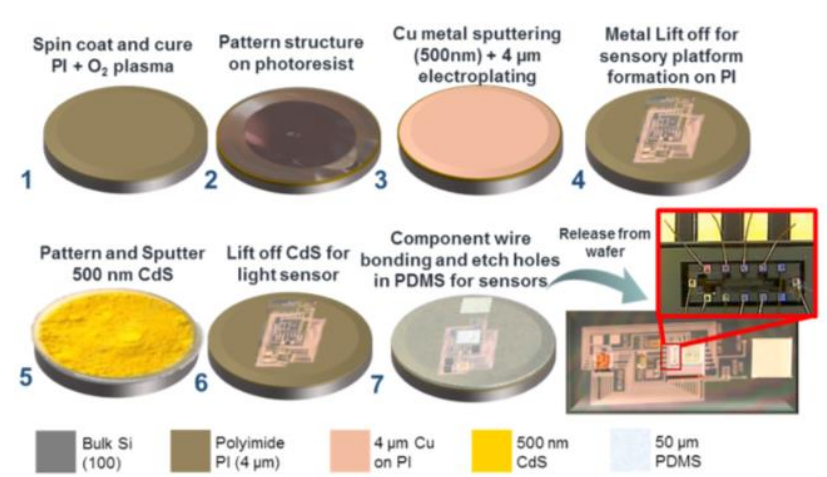

Fig. 1. 1: Spin PI on Si wafer. 2: Spin photoresist and pattern the sensory platform. 3: Deposit $500 \mathrm{~nm}$ of $\mathrm{Cu}$ using sputtering, and do electroplating to $4 \mu \mathrm{m}$ so that the film is thick enough for wire bonding of components. 4: Perform lift off to get the temperature, humidity sensors and interconnect layer. 5: Further deposit CdS light sensor on patterned photoresist. 6: Lift off for CdS light sensor formation 7: Spin $50 \mu \mathrm{m}$ PDMS for encapsulation and etch areas on the sensors that need exposure to surroundings. Finally, release the structure from the wafer by peeling off. A zoomed in portion of the image showing how the wire bonding is done from wafer to metal pad on substrate. (Reproduced with permission from Copyright (C) 2018, IEEE)

from the dropped sensors at regular intervals to provide farmers with heat, light and humidity maps of their crop fields. A small BLE chip in each platform relays data wirelessly to the drone that can then upload the collected data from all the deployed sensors to web platforms for integration into IoT systems.

\section{FLEXIBLE MICROCLIMATE SENSORY PLATFORMS}

\section{A. Ultra-lightweight flexible sensory leaf platform}

The process of photosynthesis that produces sugars for plants is necessary for plant growth. An ideal photosynthesis process requires optimal conditions of light, temperature, and humidity. As discussed, current techniques are unable to record the microclimate conditions actually experienced by the plant that contribute to its growth. Low light has a negative impact on enzyme activity and stomatal conductance, thereby limiting plants water uptake potential [13]. Low atmospheric humidity levels and elevated temperature around leaves causes plant water stress resulting in a reduced growth rate [14]. We have demonstrated that the climatic conditions differ significantly from the soil adjacent to the plant compared to the leaves of the plant $[8,15]$. The differences between soil and plant humidity, temperature and light levels become prominent when the plants are watered. The high moisture level of soil after watering increases the humidity levels and cools down the temperature at the base of the plant. A sensor placed near the soil thus records completely different readings. The amount of light seen by sensors in the soil is different from the light seen by the leaves due to the shadows cast by the plant itself. Furthermore, for non-wet conditions, soil absorbs and retains heat more than the air. The soil gets heated up more and releases heat, which is recorded by the soil sensors. These readings do not accurately represent the microclimate around the plant itself. We hereby emphasize the importance of monitoring the microclimatic conditions for plant growth from the plant leaf itself.

We have produced a sensory platform laden with flexible temperature, light, and humidity sensors on a thin flexible a
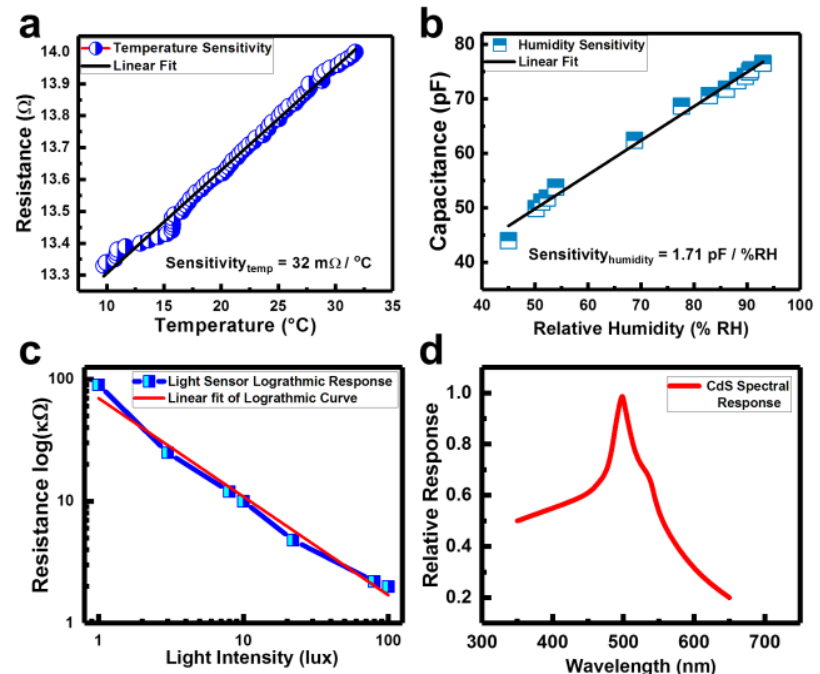

d

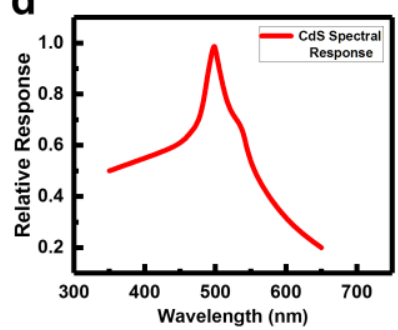

Fig. 2. (a) Temperature sensor characterization plot of resistance against temperature. (b) Humidity sensor characterization plot of capacitance sensor against RH. (c) Light sensor characterization plot of logarithmic resistance against light intensity. (d) Spectral response of CdS light senor. (Reproduced with permission from Copyright @ 2018 , IEEE)

transparent platform. The flexible substrate allows the platform to conform to the contours of the plant or the leaf, thus allowing a seamless integration, while the transparent substrate allows most of the sunlight to pass through so that the platform does not inhibit the plant growth. A specially designed Application Specific Integrated Circuit (ASIC) acquires and processes data from the sensors and transfers it to a microcontroller unit (MCU). The MCU undertake desired actions based on the recorded data. We used bare dies of all the components (ASIC, $\mathrm{MCU}$, and battery) to keep the weight to a mere 0.44 grams all housed in a compact size substrate $\left(3 \times 1.5 \mathrm{~cm}^{2}\right)$.

The temperature and humidity sensors are made of thin films of Copper $(\mathrm{Cu})$ metal on a flexible and transparent Polyimide substrate. The fabrication flow is demonstrated in Fig. 1. The thin film $\mathrm{Cu}$ metal acts as a Temperature-dependent resistor, which provided a sensitivity of $32 \mathrm{~m} \Omega /{ }^{\circ} \mathrm{C}$ and a resolution of $0.40{ }^{\circ} \mathrm{C}$ (Fig. 2a). This resolution is sufficient to monitor temperature changes as low as half a degree centigrade. The underlying Polyimide acts as the humidity sensing film for the interdigitated pattern of $\mathrm{Cu}$ metal. Polyimide has long-term stability, low manufacturing cost, integration with CMOS based process, high mechanical strength and high sensitivity to humidity. As a result, we get a linear response from humidity sensors with a sensitivity of $1.71 \mathrm{pF} / \% \mathrm{RH}$ recorded as the highest sensitivity among plant-based sensors (Fig. 2b) [8]. Studies show that chlorophyll in plants absorbs light in the range of 300-550 nm (violet to blue). Cadmium Sulphide (CdS) is sensitive to violet and blue-green light of the visible spectrum. We chose CdS as our light-sensing film deposited via sputtering. The resistance of $\mathrm{CdS}$ changes in response to varying light (Fig. 2c). It can be seen that the sensors have a strong relative response of $>0.5$ to light in the range of 350-550 $\mathrm{nm}$ (Fig. 2d). The electronics components are placed in their respective positions and wire bonded to the connections on the underlying substrate The platform is then encapsulated with PDMS. PDMS is transparent and provides mechanical strength 


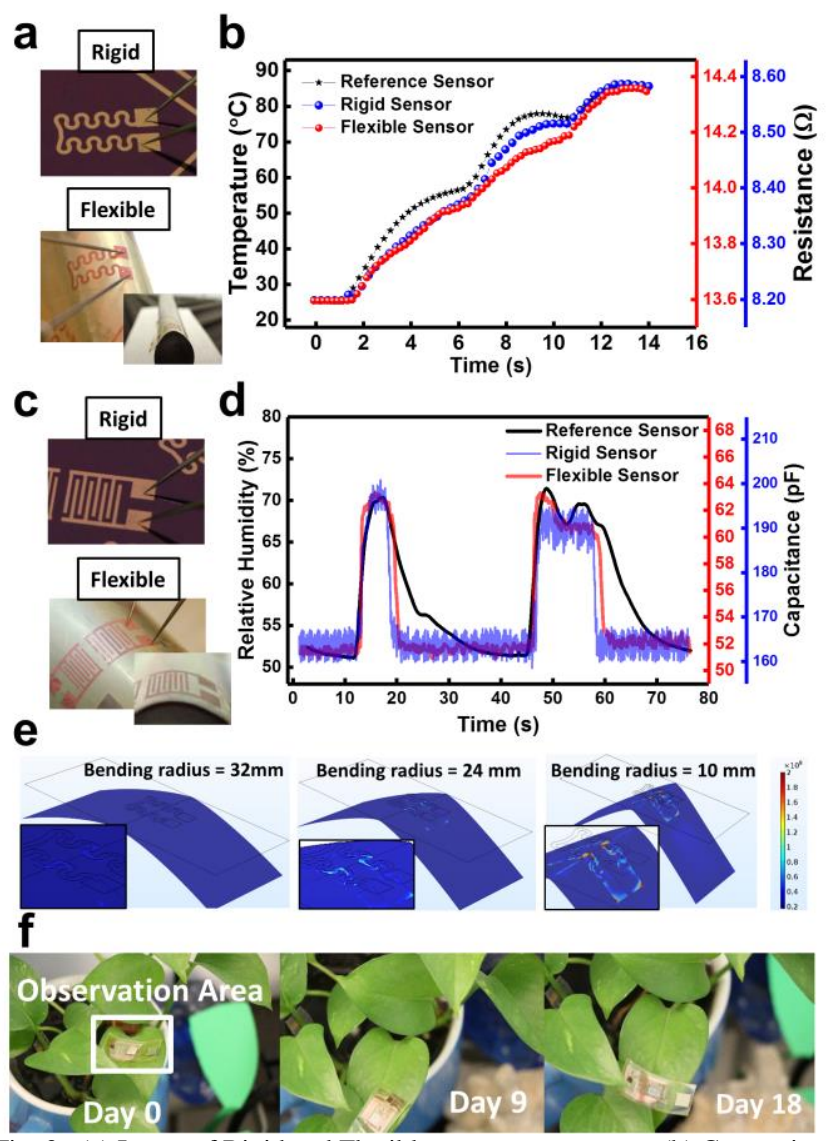

Fig. 3. (a) Image of Rigid and Flexible temperature sensor. (b) Comparison of temperature sensor performance. (c) Image of Rigid and Flexible humidity sensor. (d) Comparison of humidity sensor performance (e) Stress in flexible sensor at different bending radii. (f) Effect of the sensor patch on the photosynthetic functions of a leaf over 18 days. (Reproduced with permission from Copyright $(\odot)$ 2018, IEEE)

to the platform. Holes are etched on top of the humidity sensor using RIE to allow the moisture to reach the humidity sensor.

The bending performance of the sensors was evaluated by drawing a comparison to similar rigid sensors. The temperature sensor showed a similar response compared to its rigid counterpart for bending radius similar to the radius of a typical leaf. The response of our flexible sensors is plotted against the rigid sensor and a reference commercial sensor, where all three sensors are subjected to the same heating conditions in Figs. 3ab. Similarly, the humidity sensors were evaluated in similar conditions. The flexible sensor showed a faster response in comparison to the reference and the rigid sensor. The humidity sensing polyimide film has a faster absorption and desorption rate than the films used in rigid and reference sensors (Figs. 3cd). In order to determine the stress generated at the point of bending, we ran a Finite Element Method (FEM) simulation of a thin $500 \mathrm{~nm} \mathrm{Cu}$ film on polyimide. The model was bent to various bending radii (Fig. 3e). Minute stress is generated in response to bending stress up to $24 \mathrm{~mm}$, which is the typical bending radius to be experienced while being placed on a plant leaf. The $\mathrm{Cu}$ film reached the fracture stress at a high bending radius of $10 \mathrm{~mm}$. Lastly, in order to verify the effects of the platform on the plant, we placed it on a plant leaf for 18 days.
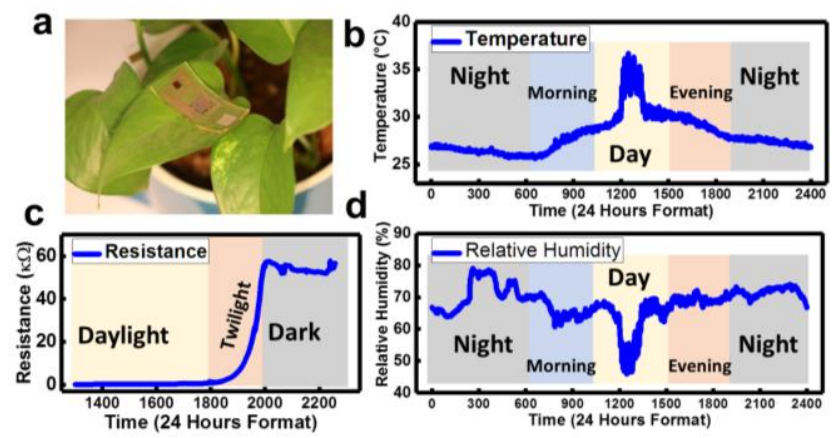

Fig. 4. (a) The sensory platform placed on the leaf of a plant. (b) Temperature monitoring for 24 hours using the sensory platform around the leaf. Temperature increases in the morning and peaks at 1200 hours daytime. (c) Light monitoring from daytime until night using the $\mathrm{CdS}$ based light sensor. During daytime the resistance is very low in the range of 1 $\mathrm{kOhm}$ and starts to linearly increase around sunset to a maximum value of $\sim 50 \mathrm{kOhm}$ at night. (d) Humidity monitoring for 24 hours using the sensory platform around the leaf. During daytime when sunlight falls on the sensor humidity levels decrease due to evaporation of water vapors. (Reproduced with permission from Copyright @ 2018, IEEE)

The results show no adverse effect on the growth and function of the plant since it is lightweight and transparent (Fig. 3f).

Consequently, the platform was tested by placing the platform on a leaf for 24 hours (Fig. 4a). Data was logged at 10 seconds intervals. The temperature logged through the day showed the rise in temperature during the day peaking around noontime (Fig. 4b). The humidity levels were recorded to be the highest during the night and reduced during the day due to the intense heat (Fig. 4c.). The data from the light sensors showed an eventual increase in resistance due to the reducing light conditions from daylight to dark (Fig. 4d). A linear response is observed as the light continues to decrease during twilight. Low light, high levels of humidity and temperature can result in an improperly growing crop. This platform can become an essential tool for plant scientists and farmers alike to find out the contributing factors towards their crop yield and adopt corrective measures to increases the output from crops.

\section{B. Flexible lightweight plant growth monitoring sensor}

Botanists and plant biologists could benefit greatly from the use of plant growth sensors. A growth sensor complemented by the information from the multisensory platform can give further in-depth information about the factors affecting the growth rate of plants. Most often the growth is monitored using advanced cameras and image processing algorithms. Such techniques use expensive equipment, specialized setups which make it hard for farmers and scientists to replicate or reproduce results [16-18]. Moreover, they do not provide quantitative information about the micrometer level growth rate of the plant. Few attempts have been made to monitor plant growth using strain sensors. One study used chitosan-based water ink to measure the expansion of fruits instead of measuring the plant growth itself [19]. Since the sensor is printed on the fruit, the surface roughness and texture change would result in an inaccurate reading of fruit growth. We have worked on a strain sensor made from thin, lightweight and flexible materials that can measure the growth of a plant at a micrometer level [9]. It can be seamlessly attached to the stem of the plant where the 


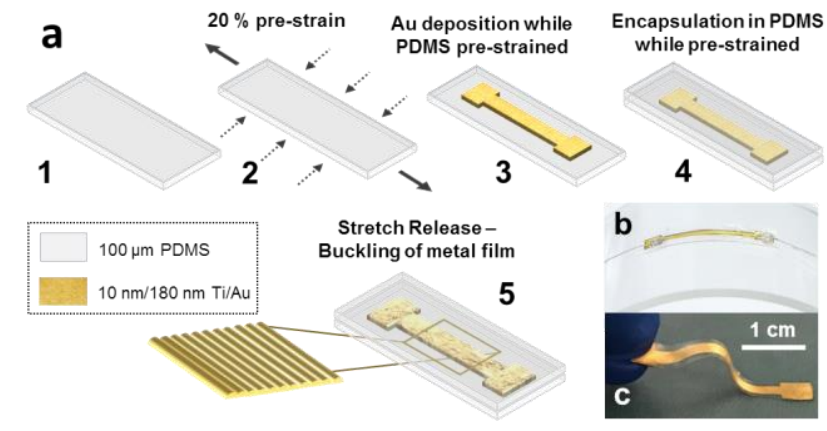

Fig. 5. (a) 1: Spin PDMS. 2: Pre-strain the PDMS layer by stretching it 20\% and sticking it on a wafer. 3: Deposit 500nm of gold film. 4: Encapsulate with another layer of PDMS. 5: Release the structure. (b) The sensor on a bent platform. (c) Sensor held in hand in bent form. (Reproduced with permission from Creative Commons Attribution 4.0 International License )

actual growth takes place. As the plant continues to grow the upper part of the stem keeps elongating [20]. The high level of sensors' strain sensitivity is achieved by the unique fabrication flow as detailed in Fig 5a. Metals are prone to developing fractures upon strain due to their high value of Young's Modulus. A 500nm thin gold film undergoes linear elongation only until $2 \%$ strain, eventually develops fractures, and breaks at $5 \%$ strain. We utilize a buckling technique in which the substrate (PDMS) is pre-stretched and then deposited with a thin film of gold as shown in Fig. 5a. Upon releasing the structure, we get a wavy structure that has an enhanced stretchability due to the buckles, before the metal film develops fractures. The sensor is coated with a second layer of PDMS before releasing to encapsulate the metal film and reduce hysteresis, as the top film provides mechanical elastic strength to the sensor to regain its original form after elongation. The process releases the residual stress from substrates and increases the theoretical strain of the metal film [9]. Buckled structures have continuous grain like structure while flat films have randomly arranged microcracks [21]. Buckled structures provide linearity to an extended range. The flexible lightweight sensor can be seen in Figs. 5b-c. Being thin and lightweight allows it to stretch easily without putting a copious strain on the stem of the plant. In order to test its performance, the sensor was placed on a stretching platform where the sensor was stretched by $1 \mathrm{~mm}$ and resulting resistance was measured using a Digital Multimeter. The results in Figs. 6a-b show that we have enhanced the linear strain range of the gold film from $2 \%$ to $22 \%$ by the use of this buckling technique. A gauge factor of 3.9 is sufficient to detect micrometer elongations of plants.

To test the sensor, we attached it on the stem of a Barley Plant at the Greenhouse facility in KAUST. The changes in resistance were monitored using a logging multimeter. In Fig. $6 \mathrm{c}$, it can be seen that the sensor is attached at the stem, which is the point of elongation during growth. The first point of the anchor is the main stem while the second point is on the flag leaf, which is in the process of elongation. The test was run for 3 hours with a sampling rate of 3 seconds. A total elongation of $284.7 \mu \mathrm{m}$ was observed over the entire time period resulting in a growth rate of $2.7 \mathrm{~cm} /$ day (Fig. $6 \mathrm{~d}$ ). Consequently, the sensor was attached to a lucky bamboo as seen in Fig. 6e. The bamboo was kept in an office area and observed for 24 hours. The results
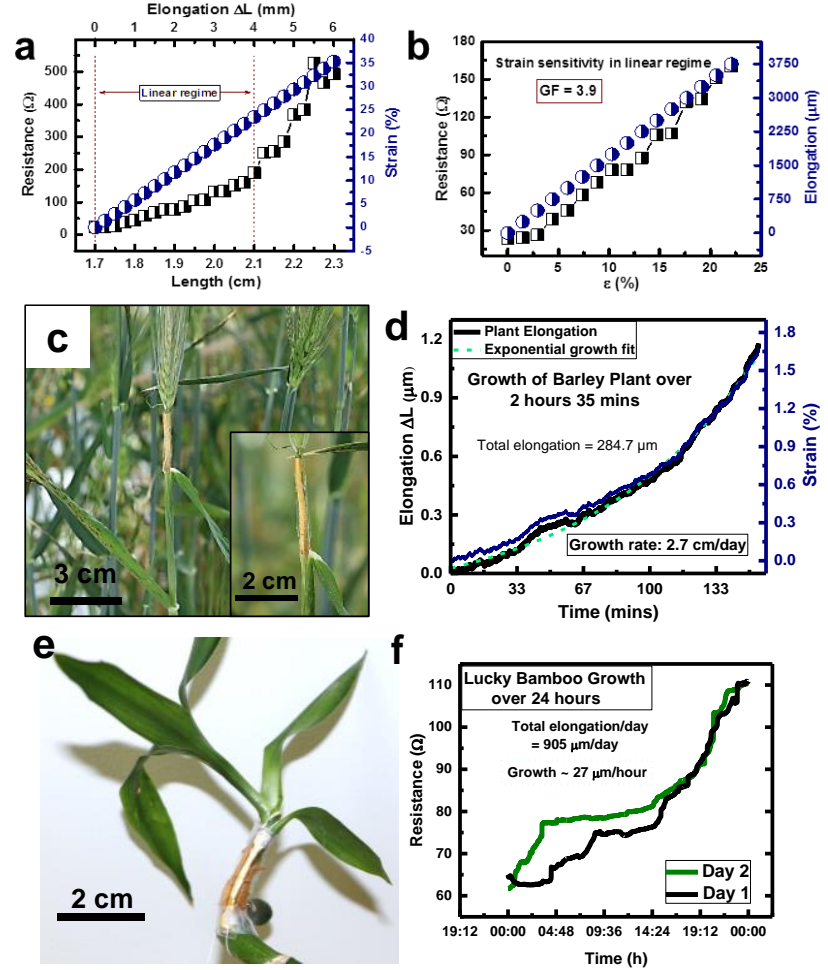

Fig. 6. (a) Plot showing the resistance increase as the strain sensor is stretched and elongated in a tensile manner, with a corresponding plot (in blue) relating elongation to tensile strain induced on the sensor. (b) Strain sensor sensitivity plot displaying a linear behavior up to $22 \%$ strain applied, corresponding to $3.75 \mathrm{~mm}$ elongation in the sensor's structure. (c) Digital photographs depicting the integration of the strain sensor system on a Barley plant for growth monitoring. (d) Plot illustrating the Barley plant growth in real-time through leaf elongation tracking for a period of 2 hours and 35 mins. (e) Digital photograph displaying the seamless integration of the growth strain sensors on the stem of a Lucky Bamboo, in between the internodes. (f) Quantitative and real-time tracking of Lucky Bamboo growth rate over a period of 24 hours continuously for 2 days. (Reproduced with permission from Creative Commons Attribution 4.0 International License)

showed a growth rate of $905 \mu \mathrm{m} /$ day (Fig. 6f). Such sensors can be a boon for both botanists and farmers who aim to study the effect of environmental factors on the growth of the plant. Moving forward, CMOS enabled microfluidic devices placed on plant leaves can monitor the plant physiology itself besides monitoring the ambient atmosphere around the plants [22].

\section{LARGE ScAled REMOte SENSING SENSORS}

We have worked extensively on sensory stems that can monitor plant growth in a significantly efficient way. In order to move forward with their integration in IoT based systems, we have directed efforts towards large-scale deployment of climate monitoring systems to provide a framework for the large-scale remote accurate macroclimate sensing in the vast areas of cultivated land. This study aims at getting maps of heat, light, and humidity over large areas of cultivated lands enabling farmers to monitor the climatic conditions in real time that affect their crop growth.

\section{A. Macro-climate monitoring sensors}

First, the microclimate sensors are developed on a thin, flexible, and transparent platform in the shape of a butterfly to 


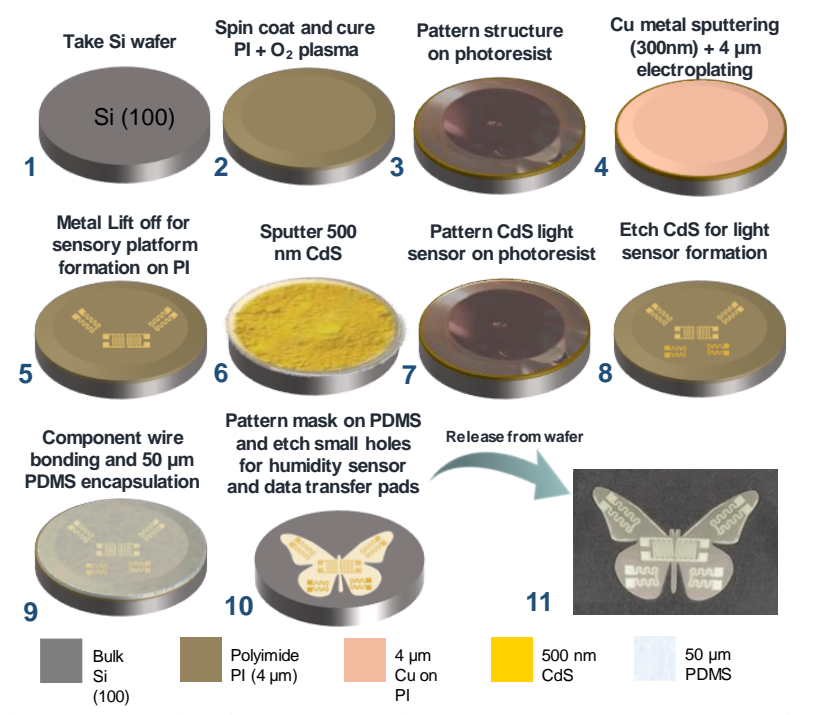

Fig. 7. 1: Take Si wafer. 2: Spin Polyimide. 3: Pattern sensors. 4: Deposit $\mathrm{Cu}$ metal and electroplate. 5: Perform lift off to form temperature and humidity sensors. 6: Sputter CdS. 7: Pattern the light sensor pattern. 8: Etch CdS for light sensor formation. 9: Spin PDMS to encapsulate sensors and form holes for humidity sensor. 10: Laser cut the sensor in the shape of butterfly. 11: Digital photograph of the sensor released from the wafer.

resonate aesthetics with the targeted application. The sensor would be deployed from air using drones but there is an equal probability of the sensor landing on either side of the platform. Thus we have developed a strategy to integrate symmetric sensors on both sides of the butterfly wings so that no matter which side the platform lands on the ground, at least one set of sensors will face the intended environment. The fabrication flow of the sensors is depicted in Fig. 7. The performance of these sensors is similar to the sensor performance shown in Fig. 2 , since the same dimensions are used to form the sensors.

\section{B. Winged Sensor Platform}

For large-scale deployment in crops, a twin blade parachute is designed using a biodegradable paper as illustrated in Figs. $8 \mathrm{a}-\mathrm{c}$. The butterfly sensor is wrapped around a small $105 \mathrm{mAh}$ Lithium polymer battery and the resulting assembly is attached to the bottom part of the parachute. The lightweight and flexible nature of the platform allows it to be seamlessly wrapped around the battery, In Fig. 8d we can see the front side of the sensor where the left wing of the butterfly platform can be seen housing the light, temperature, and humidity sensors. The inset shows a zoomed-in image where thin flexible wires can be seen that connect the sensors with the electronic system. Fig. $8 \mathrm{f}$ shows the backside of the assembly where the other set of sensors on the right wing of the butterfly can be seen. Having sensors on both sides guarantees that even if the platform lands in such a way that one side is on the ground, the sensors on the other side start recording data. In order to determine which side is facing up, sensor values from both sides are compared by the electronic interface and the side that shows more light is determined as the top side. The electronic system compromises of a Bluetooth PSOC that has integrated Capacitance-to-Digital convertor and Analog-to-Digital converter that is used to get data from the capacitance based humidity sensors, and resistance based temperature and light sensor respectively. The Bluetooth Low Energy (BLE) Programmable System on Chip (PSoC) and a

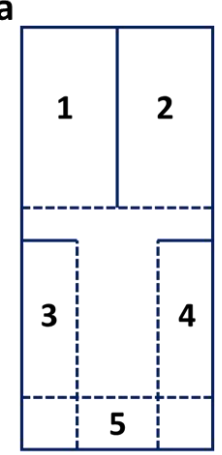

b

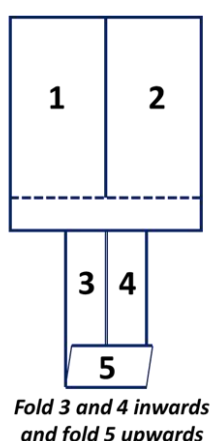

and fold 5 upwards
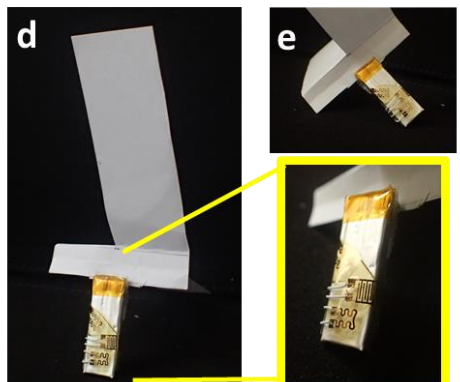

C

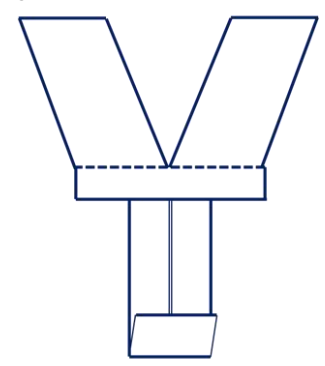

Separate 1 and 2 to form the propeller blades

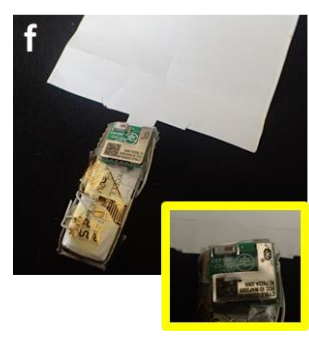

Fig. 8. (a-c) The process flow for the formation of winged parachute. (d) Butterfly sensors wrapped around the battery (front side). (e) Digital photograph of front side of platform. (f) The platform with BLE PSOC and other half of sensors (back side).

battery terminals are further encapsulated with the PDMS layer (inset image in Fig. 8f). The encapsulation avoids damage to the electronics and ensures continued operation during rain or crop irrigation while reducing the chances of contamination from the battery to the crops. Furthermore, the biodegradable paper decomposes when it comes in contact with water over time leaving behind the sensor assembly. Before the harvest season, the drone can be used to pinpoint locations of all the sensors and manually retrieve them if there is are risks involved due to the presence of the batteries in the crops. The low power BLE PSOC utilizes $8 \mathrm{~mA}$ in transmission mode and a mere $5 \mu \mathrm{A}$ in sleep mode. As a part of the deployment strategy, the sensors are preprogrammed to wake up in a fixed period in which the drone will hover over the field to collect data while being in sleep mode the rest of the time to save battery. Since the sensor relays data only when the drone flies overhead to collect the data, the battery can last for 3 months on a single charge. Generally, a crop season lasts between 3-4 months. The long battery life ensures that onetime deployment of sensors is enough for a crop season. The compact size (1.1 x $4 \mathrm{~cm}^{2}$ - without wings) and low weight of the assembly (5 gm) does not impart a negative impact on the plant growth or function even if it lands on the plant leaf.

\section{Large-scale deployment mechanism}

We formed a low profile and minimalistic 3D printed dropping mechanism using only a single servo motor (Fig. 9a). The arrangement makes smart use of a set of rack and pinion gears. The rack gear moves inside a slot. When the rack gear is at its starting position towards far left, the slot is empty. The sensory platforms are loaded in line in this slot. A small piece of Styrofoam is wrapped around the bottom part of the battery so that the battery can snugly fit in the slot (Fig. 9b). Multiple sensors are arranged in adjacent places in the slot $5 \mathrm{~mm}$ apart. 

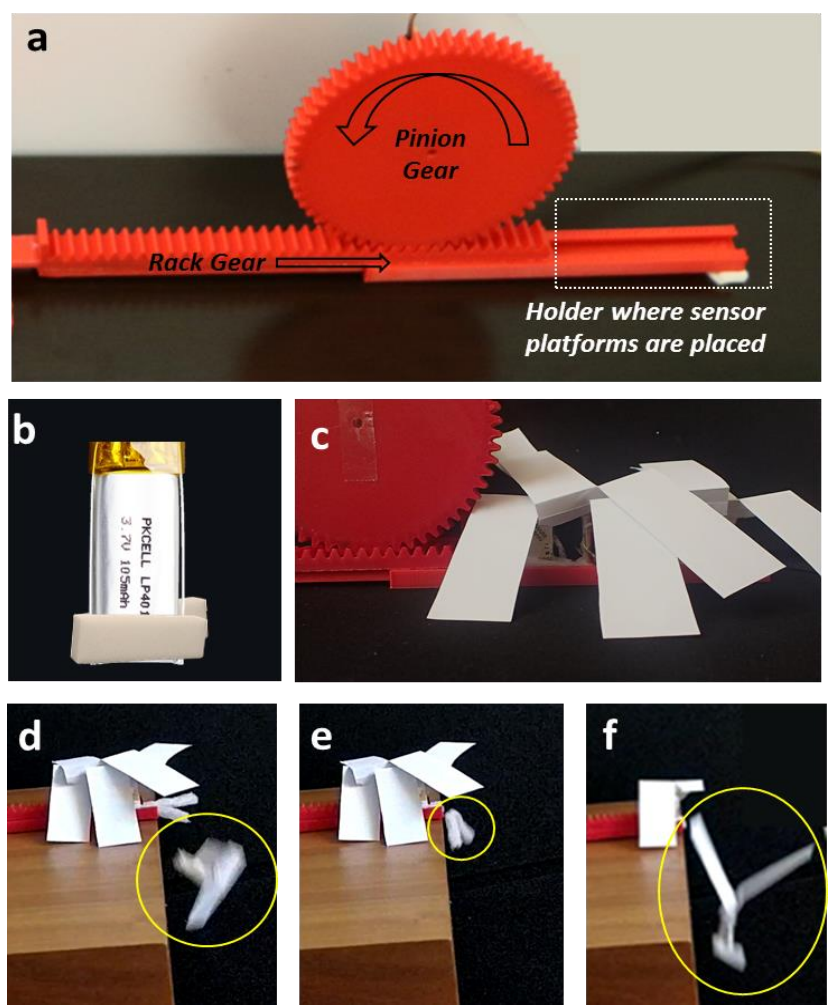

Fig. 9. (a) Dropping mechanism arrangement. (b) Styrofoam wrapped around lower part of battery. (c) Multiple sensors placed adjacently in line. (d) The platform is released, (e) followed by the Styrofoam holder. (f) The wings open up to soften the landing of the platform.

(Fig. 9c). As the pinion gear rotates anti-clockwise, the rack gear moves towards the right pushing out one platform at a time (Fig. 9d). The Styrofoam used to hold the battery inside the slot follows after the platform to be eventually discarded (Fig. 9e). The length of the dropping mechanism can be increased to house more sensors limited only by the dimensions of the carrier drone. The mechanism is only $1 \mathrm{~cm}$ wide thus allowing easily installing on even small drones. The position servo motor attached to pinion gear is controlled by the microcontroller to rotate each time such that the rack gear translates $5 \mathrm{~mm}$. This results in the release of a single platform at a time. When installed on the drone, this platform will release sensors in designated places in a crop field. The wings open up once the platform is released from the dropping mechanism ensuring a soft landing that does not damage the platform or the plant underneath (Fig. 9f).

\section{CONCLUSION}

We have previously shown high performing sensors in a lightweight platform that can be placed on a plant leaf to monitor the microclimate around the plant and provided evidence that the actual climatic conditions affecting plant growth can only be measured from the leaves [8]. Furthermore, we demonstrated a strain sensor fabricated using a unique strategy that results in 10 folds increased linear strain range to monitor plant growth at a micrometer scale for long time intervals [9]. This study extends the study by providing a framework for large-scale deployment strategy for the high performing plant monitoring sensors in vast areas of cultivated lands using drones. The flexible sensors are converted to lightweight platforms equipped with wings made from biodegradable paper to soften the landing of the platform.
A minimalistic compact dropping mechanism demonstrated the process of releasing sensors one at a time. In future, the mechanism installed on a drone will be used to release and collect data from sensors in a vast cultivated land to form the light, humidity, and heat map in order to monitor crop growth.

\section{REFERENCES}

[1] T. C. Paulitz and R. R. Bélanger, "Biological control in greenhouse systems," Annual review of phytopathology, vol. 39, no. 1, pp. 103-133, 2001. [2] L. Nhamo, R. van Dijk, J. Magidi, D. Wiberg, and K. Tshikolomo, "Improving the Accuracy of Remotely Sensed Irrigated Areas Using PostClassification Enhancement Through UAV Capability," Remote Sensing, vol. 10 , no. 5 , p. $712,2018$.

[3] P. Link. (June 2017). Plant Link. Available: http://myplantlink.com/

[4] P. F. Power. (June 2017). Parrot Flower Power. Available: http://global.parrot.com/au/products/flower-power/

[5] C.-T. Chiang and J.-X. Lin, "A CMOS digitized monolithic sun sensor transducer with calibration circuits for monitoring solar radiation of tomato crops," in SENSORS, 2015 IEEE, 2015, pp. 1-4: IEEE.

[6] G. Hornero, J. E. Gaitán-Pitre, E. Serrano-Finetti, O. Casas, and R. PallasAreny, "A novel low-cost smart leaf wetness sensor," Computers and Electronics in Agriculture, vol. 143, pp. 286-292, 2017.

[7] J. M. Aaslyng, E. Rosenqvist, and K. Høgh-Schmidt, "A sensor for microclimatic measurement of photosynthetically active radiation in a plant canopy," Agricultural and Forest Meteorology, vol. 96, no. 4, pp. 189-197, 1999.

[8] S. M. Khan, S. F. Shaikh, N. Qaiser, and M. M. Hussain, "Flexible Lightweight CMOS-Enabled Multisensory Platform for Plant Microclimate Monitoring," IEEE Transactions on Electron Devices, vol. 65, no. 11, pp. 50385044, 2018.

[9] J. M. Nassar, S. M. Khan, D. R. Villalva, M. M. Nour, A. S. Almuslem, and M. M. Hussain, "Compliant plant wearables for localized microclimate and plant growth monitoring," npj Flexible Electronics, vol. 2, no. 1, p. 24, 2018.

[10] J. M. Nassar et al., "Compliant lightweight non-invasive standalone "Marine Skin" tagging system," npj Flexible Electronics, vol. 2, no. 1, p. 13, 2018.

[11] S. M. Khan, N. Qaiser, and M. M. Hussain, "Do-It-Yourself (DIY) based Flexible Paper Sensor Based Electronic System for Pill Health Monitoring," in 2018 International Flexible Electronics Technology Conference (IFETC), 2018, pp. 1-2: IEEE.

[12] S. F. Shaikh et al., "Noninvasive Featherlight Wearable Compliant “ Marine Skin ”: Standalone Multisensory System for Deep - Sea Environmental Monitoring," Small, p. 1804385, 2019.

[13] G. D. Farquhar and T. D. Sharkey, "Stomatal conductance and photosynthesis," Annual review of plant physiology, vol. 33, no. 1, pp. 317$345,1982$.

[14] R. Grange and D. Hand, "A review of the effects of atmospheric humidity on the growth of horticultural crops," Journal of Horticultural Science, vol. 62, no. 2, pp. 125-134, 1987.

[15] J. M. Nassar, S. M. Khan, M. Nour, A. Almuslem, and M. M. Hussain, "Plant Wearable for Enhanced Agricultural Productivity," in Meeting Abstracts, 2018, no. 26, pp. 1574-1574: The Electrochemical Society.

[16] B. Alberton et al., "Introducing digital cameras to monitor plant phenology in the tropics: applications for conservation," Perspectives in Ecology and Conservation, vol. 15, no. 2, pp. 82-90, 2017.

[17] M. Ulman, "Active imaging systems for plant growth monitoring," ed: Google Patents, 2016.

[18] R. Meng et al., "Growth curve registration for evaluating salinity tolerance in barley," Plant methods, vol. 13, no. 1, p. 18, 2017.

[19] W. Tang, T. Yan, J. Ping, J. Wu, and Y. Ying, "Rapid Fabrication of Flexible and Stretchable Strain Sensor by Chitosan - Based Water Ink for Plants Growth Monitoring," Advanced Materials Technologies, 2017.

[20] B. Junction. (September 2017). Plant Structure Bi. Available: http://www.biologyjunction.com/plant_structure_bi1.htm

[21] H. Hocheng and C.-M. Chen, "Design, fabrication and failure analysis of stretchable electrical routings," Sensors, vol. 14, no. 7, pp. 11855-11877, 2014. [22] S. M. Khan, A. Gumus, J. M. Nassar, and M. M. Hussain, "CMOS enabled microfluidic systems for healthcare based applications," Advanced Materials, vol. 30 , no. 16 , p. $1705759,2018$. 